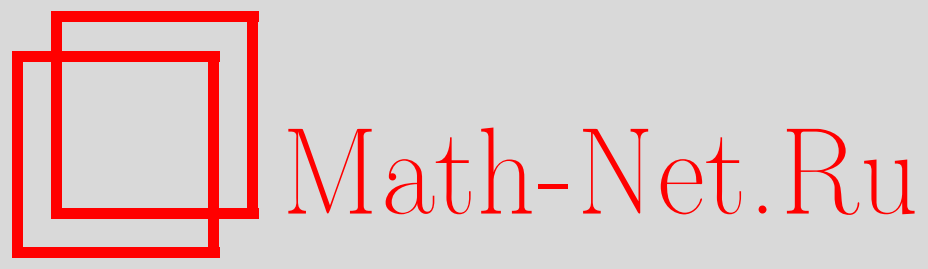

С. В. Дужин, М. В. Карев, Определение ориентации струнных зацеплений при помощи инвариантов конечного типа, Функи. анализ и его прил., 2007, том 41, выпуск 3, 48-59

DOI: https://doi.org/10.4213/faa2865

Использование Общероссийского математического портала MathNet.Ru подразумевает, что вы прочитали и согласны с пользовательским соглашением http://www . mathnet.ru/rus/agreement

Параметры загрузки:

IP : 54.84 .234 .179

26 апреля 2023 г., $17: 20: 36$

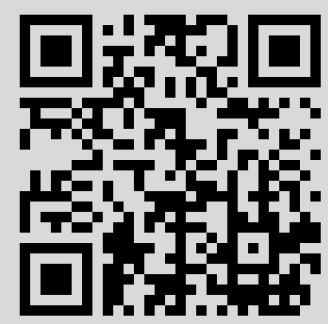


Функционалъный анализ и его приложения

2007, т. 41, вып. 3, с. 48-59

УДК 515.16

\title{
Определение ориентации струнных зацеплений при помощи инвариантов конечного типа*
}

\author{
(c) 2007. С. В. Дужин, М. В. КАРев
}

\section{§1. Введение}

Хорошо известно, что классические инвариантные полиномы (Джонса, HOMFLY и т. д.) и вообе квантовые инварианты узлов принимают одинаковые значения на узлах, отличающихся ориентацией. Класс инвариантов Васильева строго шире [16], и вопрос о том, могут ли инварианты Васильева различать ориентацию, по сей день не решен. В этой работе мы изучаем соответствующую проблему для зацеплений с более чем одной компонентой.

Пусть $S_{p}^{1}$ - дизъюнктное объединение $p$ пронумерованных экземпляров ориентированной окружности, $\mathbb{R}_{p}^{1}$ - дизъюнктное объединение $p$ пронумерованных экземпляров вещественной прямой, а $I_{p}^{1}$ - дизъюнктное объединение $p$ пронумерованных ориентированных отрезков [0,1].

Определение. Назовем замкнутым р-компонентным заиеплением гладкое вложение многообразия $S_{p}^{1}$ в ориентированное трехмерное пространство $\mathbb{R}^{3}$, рассматриваемое с точностью до изотопии, сохраняющей компоненты.

Длинным р-компонентным заиеплением называется гладкое вложение многообразия $R_{p}^{1}$ в ориентированное трехмерное пространство $\mathbb{R}^{3}$ с фиксированной асимптотикой на бесконечности: $x_{i}(t)=[i, 0, t]$ при $|t|>C$, рассматриваемое с точностью до изотопии, тождественной вне шара достаточно большого радиуса, и евклидова движения пространства.

Струнным р-компонентным зацеплением назовем гладкое вложение многообразия $I_{p}^{1}$ в полосу $\mathbb{R}^{2} \times[0,1] \subset \mathbb{R}^{3}$ с закрепленными концами: $x_{i}(t)=[i, 0, t]$ для $t=0,1$, рассматриваемое с точностью до изотопии, тождественной на границе, и евклидова движения пространства.

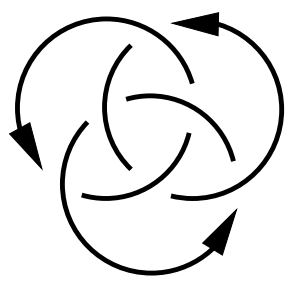

Замкнутое зацепление

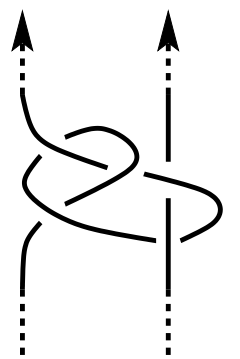

Длинное зацепление

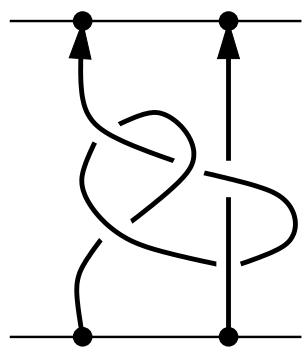

Струнное зацепление

*Работа поддержана грантами РФФИ 05-01-01012, РФФИ 05-01-00899, НШ-1972.2003.1, 06-01-72551-НЦНИЛ. 
Очевидно, что теории длинных и струнных зацеплений эквивалентны. В случае узлов $(p=1)$ обе эти теории также эквивалентны теории замкнутых зацеплений, но это становится неверным в случае $p>1$.

Bопрос об обратимости в этом случае ставится следующим образом. Пусть $L$ - некоторое зацепление и $L^{\prime}$ - его обращение, т. е. то же зацепление с той же нумерацией компонент, но с противоположной ориентацией. Зацепление $L$ называется обратимым, если $L$ и $L^{\prime}$ эквивалентны. Существуют ли необратимые зацепления? Если ответ утвердителен, то какие инварианты могут их различать?

В случае узлов этот вопрос оставался нерешенным долгое время, пока Троттер [14] в 1964 г. не доказал необратимость некоторых узлов (например, крендельного узла $\left.P_{3,5,7}\right)$. Простейшим необратимым узлом является узел $8_{17}([8])$. Инварианты, использованные в работах [14], [8] и др. для доказательства неэквивалентности узла своему обратному, довольно сложны, и до сих пор не известно, можно ли различить какую-то пару взаимно обратных узлов при помощи инвариантов конечного типа.

В случае зацеплений единственным опубликованным результатом по данной проблеме является теорема Лина [11], которая утверждает, что инварианты Васильева различают ориентацию замкнутых зацеплений с шестью или более компонентами. Имеется еще несколько работ, имеющих к этой задаче лишь косвенное отношение, например, Бар-Натан в [2] изучает гомотопические инварианты струнных зацеплений, а Лин [10] и Фидлер [7] используют классы инвариантов, отличные от классических инвариантов конечного типа.

Настоящая статья посвящена вопросу определения ориентации с помощью инвариантов конечного типа для струнных (или длинных) зацеплений. Переформулировка проблемы в терминах хордовых диаграмм немедленно показывает, что это возможно для числа нитей $p>2$. В случае двухкомпонентных зацеплений $(p=2)$ вопрос нетривиален; ниже мы даем доказательство следующей теоремы.

Теорема. Существует инвариант Васильева $f$ порядка, не превосходящего 7, и двухкомпонентное струнное защепление $L$, такие, что $f\left(L^{\prime}\right) \neq f(L)$.

Мы дадим два доказательства этой теоремы. Оба будут основаны на прямых вычислениях. Первое доказательство ( $\S 2,3$, предложение 1$)$ использует хордовые диаграммы и требует привлечения компьютерного счета. Второе $(\S \S 4,5$, предложение 2) использует диаграммы Якоби; необходимые для него вычисления выполняются вручную. Оба доказательства на самом деле относятся к оснащенным зацеплениям, но в 66 мы покажем, каким образом результат переносится на неоснащенный случай, что и завершит доказательство теоремы.

Вопрос об обращении ориентации струнных $p$-компонентных зацеплений на уровне инвариантов конечного типа тесно связан с вопросом о коммутативности алгебры $\mathscr{A}(p)$ хордовых диаграмм на $p$ нитях. А именно, некоммутативность алгебры $\mathscr{A}(p)$ влечет за собой существование элементов, неинвариантных относительно замены ориентации. В самом деле, операция замены ориентации $\tau$ (см. ниже) является антиавтоморфизмом. Если предположить симметричность всех элементов из $\mathscr{A}(p)$, то мы бы получили, что $x y=\tau(x y)=\tau(y) \tau(x)=y x$ для любых $x, y \in \mathscr{A}(p)$. (Мы признательны рецензенту, обратившему наше внимание на это рассуждение.) 
Некоммутативность алгебры $\mathscr{A}(p)$ при $p>2$ очевидна, а при $p=2$, хотя и считалась известной как фольклорный факт, но нигде до сих пор не была доказана. Предложение 1 настоящей работы содержит, в частности, первое строгое доказательство этого утверждения.

\section{§2. Сведение к хордовым диаграммам}

Инварианты конечного типа для различных типов зацеплений определяются так же, как и в классическом случае узлов, см. [1], [6].

Пусть $\mathbb{F}$ - поле характеристики 0, например $\mathbb{Q}$ или $\mathbb{C}$. Обозначим через $V_{n}(p)$ пространство $\mathbb{F}$-значных инвариантов Васильева для длинных $p$-компонентных зацеплений степени не более чем $n$, через $\mathscr{A}_{n}(p)$ пространство, порожденное хордовыми диаграммами степени $n$ на $p$ нитях с точностью до четырехчленных соотношений (см. [6], [13]), и через $W_{n}(p)=\mathscr{A}_{n}^{*}(p)=\operatorname{Hom}_{\mathbb{F}}\left(\mathscr{A}_{n}(p), \mathbb{F}\right)$ соответствующее пространство весовых систем. Существует линейное отображение $\sigma_{n}^{p}: V_{n}(p) \rightarrow W_{n}(p)$ (взятие символа инварианта Васильева), ядро которого совпадает с $V_{n-1}(p)$, а образ состоит из весовых систем, обращающихся в 0 на хордовых диаграммах с изолированными хордами. (Для случая оснащенных зацеплений образ совпадает с $W_{n}(p)$.) Через $\mathscr{A}(p)$ мы обозначаем прямую сумму пространств $\mathscr{A}_{n}(p)$ по всем $n \geqslant 0$. Пространство $\mathscr{A}(p)$ образует градуированную алгебру (умножение - конкатенация диаграмм).

Как и прежде, обозначим через $L^{\prime}$ обращение зацепления $L$. Полагая $\tau_{V}(f)(L)$ $=f\left(L^{\prime}\right)$ для $f \in V$, мы получаем инволюцию $\tau_{V}$ пространства инвариантов Васильева. В терминах хордовых диаграмм соответствующая операция $\tau_{A}$ действует так:
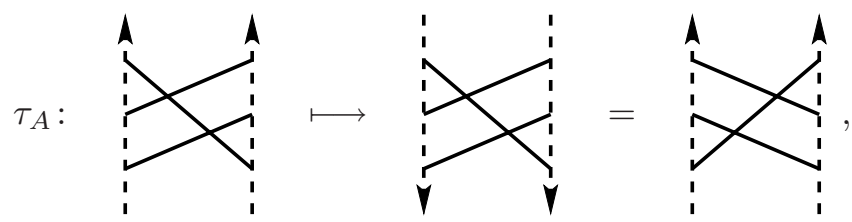

т. е. она меняет ориентацию всех компонент носителя хордовой диаграммы, или, что то же самое, отражает изображенную в плоскости диаграмму относительно горизонтальной линии, если считать, что компоненты носителя ориентированы вертикально (ориентация компонент при этом сохраняется).

То же самое пространство $\mathscr{A}(p)$ можно получить как линейную оболочку всех обобщенных хордовых диаграмм, т. е. 1-3-валентных графов, одновалентные вершины которых отождествлены с точками на многообразии $\mathbb{R}_{p}^{1}$, с циклическим порядком полуребер, сходящихся в каждой трехвалентной вершине.

Обобщенные хордовые диаграммы понимаются как линейные комбинации обычных хордовых диаграмм, получаемые применением STU-соотношений следующего вида (подробнее см. [1])
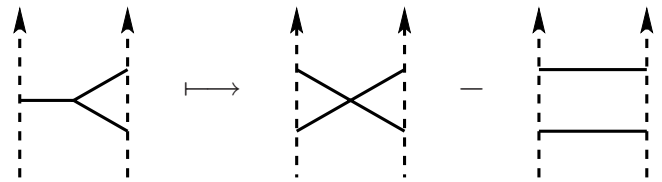

(На рисунках здесь и ниже используется такое соглашение: в каждой трехвалентной вершине ребра упорядочены против часовой стрелки.) 
Для обобщенных хордовых диаграмм определение инволюции $\tau_{A}$ несколько усложняется. Распространение операции $\tau_{A}$ на обобщенные хордовые диаграммы посредством соотношений STU приводит к следующему правилу:

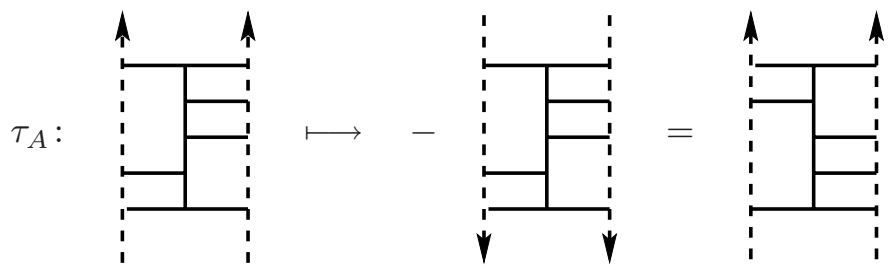

т. е. необходимо поменять ориентацию на каждой компоненте носителя и умножить результат на 11 в зависимости от четности числа трехвалентных вершин, или, что то же самое, просто отразить плоское изображение диаграммы относительно горизонтальной прямой, не меняя ориентации носителя.

Лемма 1. Инволющия $\tau_{A}$ есть градуированный аналог инволюиии $\tau_{V}$, m.e. следующая диаграмма коммутативна:

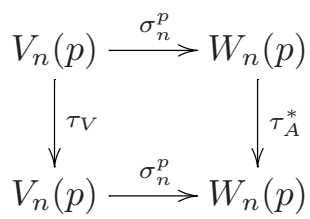

Доказательство. Для обычных хордовых диаграмм это немедленно следует из определения отображения $\sigma$ ([1], [6]). Распространение на обобщенные хордовые диаграммы происходит, как объяснено выше.

Проблема существования необратимых струнных зацеплений с помощью инвариантов конечного типа преобразуется в следующую: существует ли хордовая диаграмма на $p$ нитях, не эквивалентная своей обратной по модулю четырехчленных соотношений. При $p \geqslant 3$ соответствующий пример легко построить:
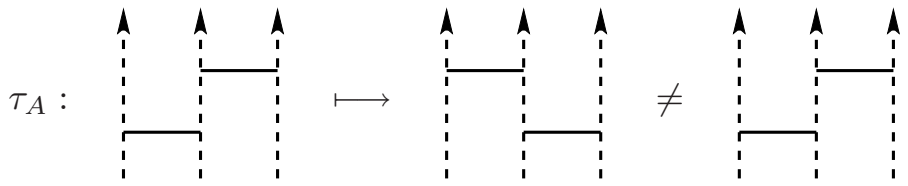

При $p=2$ проблема нетривиальна, поскольку, как легко убедиться, хордовые диаграммы малых степеней (например, диаграммы в первом отображении в настоящем параграфе) все $\tau_{A}$-инвариантны. В следующем параграфе мы предъявим диаграмму, не являющуюся $\tau_{A}$-инвариантной.

\section{§3. Первое доказательство теоремы}

Для того чтобы доказать, что некоторый элемент пространства $\mathscr{A}(p)$ отличен от нуля, естественно использовать весовые системы, т. е. линейные функционалы на этом пространстве. Одной из наиболее часто применяемых весовых систем является гомоморфизм Концевича $\varphi=\varphi_{\mathfrak{g}}: \mathscr{A}(p) \rightarrow U(\mathfrak{g})^{\otimes p}$, который строится по метризованной алгебре Ли g (см. [9], [6]). В действительности $\varphi$ принимает значения в $\mathfrak{g}$-инвариантной подалгебре $U(p)=\left[U(\mathfrak{g})^{\otimes p}\right]^{\mathfrak{g}}$. Мы дадим 
описание действия этой весовой системы для случая $\mathfrak{g}=\mathfrak{g l}_{N}$, используя стандартный базис $e_{i j}$ (матрица с 1 на месте $(i, j)$ и остальными нулями) и метрику, определенную правилом сопряжения $e_{i j}^{*}=e_{j i}$.

Лемма 2. Пусть $D \in \mathscr{A}(p)-($ обобщенная) хордовая диаграмма на $р$ нитях. Элемент $\varphi(D) \in U\left(\mathfrak{g l}_{N}\right)^{\otimes p}$ может бъть получен следующей процедурой. Возъмем альтернированную сумму по всем возможным вариантам разрешения [1] внутренних тройных точек диаграммы D. Для каждого варианта разрешения необходимо обозначить компоненты связности полученной диаграммы различными независимыми индексами, затем заменить кажсую пару сходящихся индексов на $e_{i j}$ и взять сумму по всем встречаюшимся индексам от 1 до N. (Если встречаются замкнутые компоненты, то они переходят в умножение на $N$.)

Доказательство этой леммы в точности повторяет аналогичное для случая $p=1$, которое можно найти в [1], [6]. Приведем лишь пример:
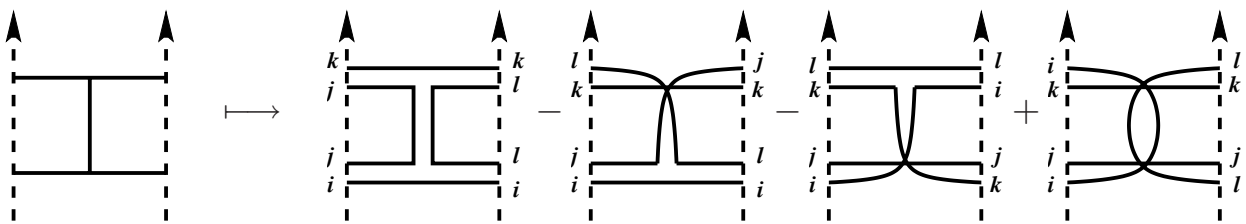

Следовательно, образ этой диаграммы при применении к ней весовой системы $\varphi$ равен

$$
\sum_{i, j, k, l=1}^{N}\left(e_{i j} e_{j k} \otimes e_{l i} e_{k l}-e_{i j} e_{k l} \otimes e_{l i} e_{j k}-e_{i j} e_{k l} \otimes e_{j k} e_{l i}+e_{i j} e_{k i} \otimes e_{j l} e_{l k}\right) .
$$

Заметим, что порядок сомножителей $e_{i j}$ согласуется с ориентацией каждой из компонент носителя, но порядок индексов $i, j$ соответствует «правилу обхода по кругу» (в нашем случае это снизу вверх на левой компоненте и сверху вниз на правой).

Обозначим через $\tau_{U}$ оператор на $U\left(\mathfrak{g l}_{N}\right)^{\otimes p}$, который обращает порядок сомножителей в каждом из мономов и меняет местами индексы у каждого базисного элемента $e_{i j}$, например $\tau_{U}\left(e_{12} e_{23} \otimes e_{13} e_{24}\right)=e_{32} e_{21} \otimes e_{42} e_{31}$. Этот оператор является инволюцией, сохраняющей ad-инвариантную часть $U(p)=\left[U\left(\mathfrak{g l} \mathfrak{l}_{N}\right)^{\otimes p}\right]^{\mathfrak{g}}$.

Следующая лемма непосредственно вытекает из конструкции отображения $\varphi$.

Лемма 3. Диаграмма

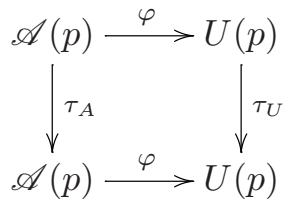

коммутативна.

Из этой леммы следует, что необратимость хордовой диаграммы может быть замечена на уровне универсальной обертывающей алгебры: если $\varphi$-образ диаграммы не $\tau_{U}$-инвариантен, то и сама диаграмма не инварианта относительно $\tau_{A}$. Таким образом, следующее предложение доказывает сформулированную в $§ 1$ теорему. 
Предложение 1. Каждая из приведенных ниже диаграмм не эквивалентна своему образу при применении инволюиии $\tau_{A}$ :
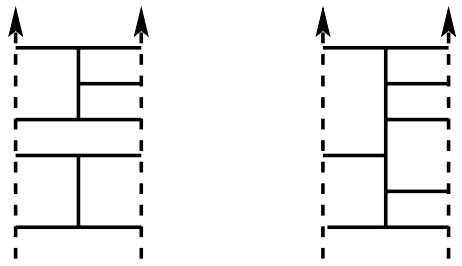

Доказательство. Вычисленные с помощью компьютера $\varphi$-образы диаграмм и их обращений различны, если в качестве алгебры Ли брать $\mathfrak{g l}_{4}$ (и, следовательно, любую алгебру $\mathfrak{g l}_{N}$ с $\left.N \geqslant 4\right)$.

Вычисления проводились так: фиксируем лексикографический порядок на множестве базисных элементов $e_{i j}$ и преобразуем выражение для $\varphi(D)$, полученное по изложенному выше алгоритму, с использованием коммутационных соотношений так, чтобы порядок сомножителей в каждом из мономов становился лексикографическим. Программы, использованные для расчетов, а также входные и выходные данные имеются на интернет-странице [5]. Например, образ диаграммы, изображенной слева, состоит из 58378 слагаемых; его вычисление занимает несколько часов с использованием современного компьютера средней мощности. Поскольку эта диаграмма является произведением двух $\tau$-симметричных диаграмм, мы заодно доказали некоммутативность алгебры $\mathscr{A}(2)$.

\section{§4. Сведение к диаграммам Якоби}

Другая, пожалуй, более простая переформулировка проблемы обратимости может быть дана в терминах крашеных диаграмм Якоби. Крашеная диаграмма Якоби - это почти то же самое, что и китайский иероглиф, определенный в [1], за исключением того, что ее одновалентные вершины помечены одним из $p$ цветов. Пространство крашеных диаграмм Якоби определяется как линейное пространство $\mathscr{B}(p)$, формально порожденное всеми $p$-цветными диаграммами Якоби и профакторизованное по соотношениям антисимметрии и IHX (см. [1], [6]).

По аналогии с теоремой 8 из [1] можно доказать, что отображение симметризации $\chi: \mathscr{B}(p) \rightarrow \mathscr{A}(p)$ является линейным изоморфизмом двух векторных пространств (см. также [12]).

Конструкцию отображения $\chi$ мы объясним на примере $p=2$. Пусть $D-$ диаграмма Якоби с $k$ «ногами» цвета 1 и $l$ «ногами» цвета 2 . Тогда $\chi(D)$ будет средним по всем $k$ ! $l$ ! способам прикрепления ног цвета 1 к первой компоненте носителя и ног цвета 2 ко второй; например,
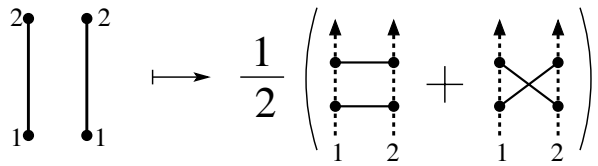

Крашеные диаграммы Якоби можно рассматривать как симметрические элементы пространства $\mathscr{A}(p)$ - ровно в том же смысле, в котором полиномы на 
пространстве $\mathfrak{g}^{*}$ отождествляются с симметрическими элементами универсальной обертывающей алгебры $U(\mathfrak{g})$ в силу теоремы Пуанкаре-Биркгофа-Витта.

Изоморфизм $\chi$ чрезвычайно полезен для наших нужд, поскольку инволюция $\tau_{A}$, перенесенная в $\mathscr{B}(p)$ с его помощью, в терминах диаграмм Якоби приобретает особенно простой вид. А именно, справедливо следующее утверждение.

Лемма 4. Пусть $\tau_{B}: \mathscr{B}(p) \rightarrow \mathscr{B}(p)$ - линейный оператор, тождественный на всех диаграммах Якоби с четным числом ног и умножсающий на -1 кажсдую диаграмму с нечетным числом ног. Тогда диаграмма

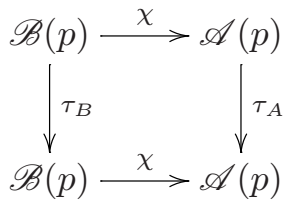

коммутативна.

Доказательство. Этот факт очевидным образом вытекает из определений отображений $\tau_{A}$ и $\tau_{B}$, данных выше. Приведем простой пример, иллюстрирующий ход рассуждений. Пусть

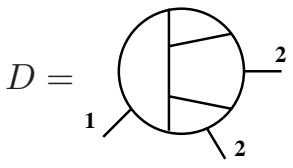

Тогда по определению

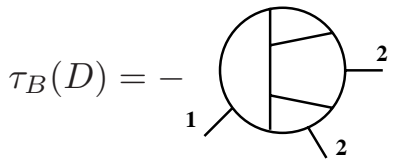

Далее,

$$
\chi(D)=\frac{1}{2}
$$

и, следовательно,

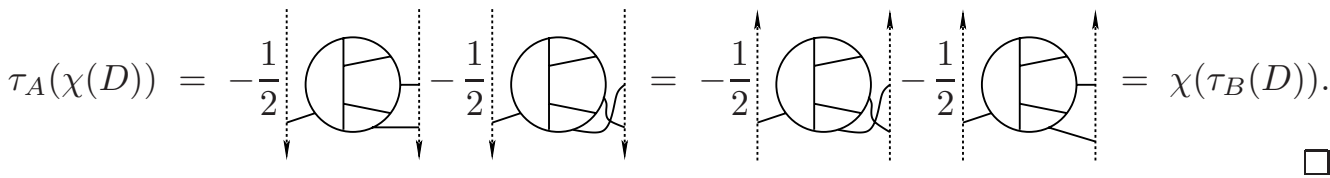

Проблема обратимости 2-компонентных струнных зацеплений с учетом приведенной выше леммы переформулируется таким образом: существует ли ненулевая двухиветная диаграмма Якоби с нечетным числом ног? В следующем параграфе мы приведем пример такой диаграммы.

\section{§5. Второе доказательство теоремы}

Возьмем метризованную алгебру Ли $\mathfrak{g}$ и обозначим через $S(\mathfrak{g})$ симметрическую алгебру векторного пространства $\mathfrak{g}$. Конструкция весовой системы $\mathscr{B}(1) \rightarrow$ 
$S(\mathfrak{g})$, описанная в [1], [6], допускает обобщение на случай произвольного значения $p$, позволяя получить гомоморфизм $\psi: \mathscr{B}(p) \rightarrow S(\mathfrak{g})^{\otimes p}$, образ которого лежит в $\mathfrak{g}$-инвариантной подалгебре $p$-й симметрической степени $S(p)=$ $\left[S(\mathfrak{g})^{\otimes p}\right]^{\mathfrak{g}}$.

Лемма 5. Отображение $\psi$ делает коммутативной следующую диаграмму:

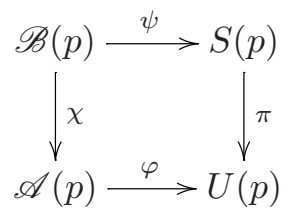

где х - изоморфизм, определенный в предыдущем параграфе, $\varphi$ - весовая система Концевича для алгебры $\mathscr{A}(p)$, a $\pi$ есть р-я тензорная степень изоморфизма Пуанкаре-Биркгофа-Витта.

Доказательство. Доказательство полностью повторяет соответствующее доказательство для случая $p=1$.

Для случая алгебры Ли $\mathfrak{g}=\mathfrak{g l}_{N}$ существует графический алгоритм нахождения $\psi$-образа, аналогичный процедуре применения оператора $\varphi$, описанной в $\S 3$. Единственное отличие состоит в том, что теперь базисные элементы $e_{i j}$ коммутируют, и мы можем не заботиться о порядке сомножителей в мономах. Приведем пример:

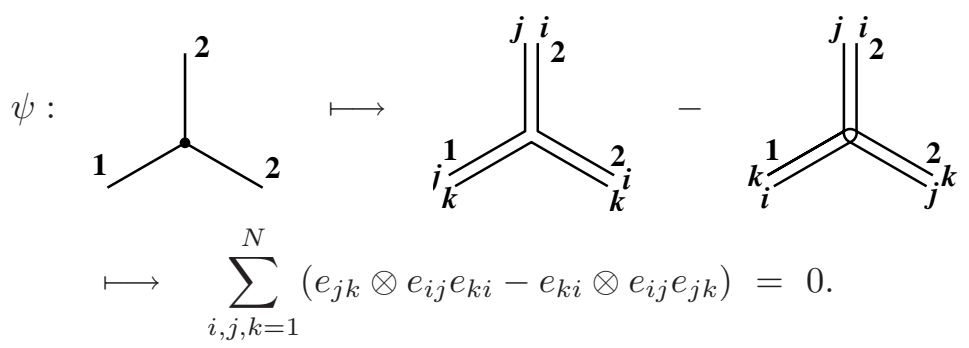

Преимущество отображения $\psi$ состоит в том, что $\mathfrak{g}$-инвариантная часть пространства $S\left(\mathfrak{g l}_{N}\right)^{\otimes p}$ обладает более прозрачной структурой по сравнению с $U\left(\mathfrak{g l}_{N}\right)^{\otimes p}$. Назовем $p$-цветным ожерелъем порядка $n$ комбинаторный объект, определенный как последовательность из $n$ чисел от 1 до $p$, рассматриваемая с точностью до циклических перестановок членов последовательности; визуализацией этого объекта являются расположенные на ориентированной окружности $n$ бусин $p$ цветов. Каждому ожерелью можно сопоставить ad-инвариантный элемент из $S\left(\mathfrak{g l}_{N}\right)^{\otimes p}$ следующим образом. Сопоставим каждой дуге ожерелья свой переменный индекс $\left(i, j\right.$ и т. д.), каждой бусине сопоставим элемент $e_{i j}$, где $i$ - индекс на входящей дуге, а $j$ - индекс на выходящей, и поставим элемент $e_{i j}$ в тензорный сомножитель, номер которого совпадает с номером цвета соответствующей бусины. Эти элементы перемножим, а потом просуммируем по всем встречающимся индексам от 1 до $N$. Например:

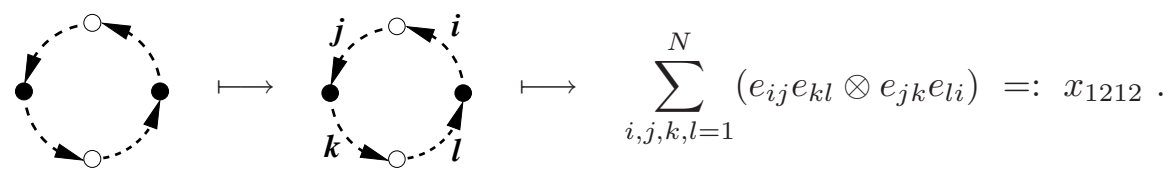


Здесь и ниже мы обозначаем элемент пространства $S\left(\mathfrak{g l}_{N}\right)^{\otimes p}$, отвечающий какому-то ожерелью, через $x_{\mu}$, где $\mu$ - последовательность цветов, лексикографически наименьшая среди всех циклических сдвигов.

Лемма 6. В случае алгебры Ли $\mathfrak{g}=\mathfrak{g l}_{N} \mathfrak{g}$-инвариантная часть пространства $S(\mathfrak{g})^{\otimes p}$ совпадает с алгеброй, порожденной всеми ожерельями. Алгебраические соотношения между элементами могут иметь место при малых $N$, но исчезают при $N \rightarrow \infty$.

Доказательство. Утверждения, близкие по формулировке к этой лемме, доказаны в [17] и [15]. Данное утверждение из них легко выводится.

Замечание. Весовую систему со значениями в алгебре ожерелий можно определить непосредственно, не апеллируя к алгебрам Ли. Мы собираемся написать об этом в отдельной публикации.

В алгебре, порожденной ожерельями, существует инволюция $\tau_{S}$, обращающая ориентацию каждого ожерелья. Для двухцветных ожерелий она действует тождественно вплоть до порядка 5; ожерелье наименьшего порядка, не инвариантное относительно $\tau_{S}$, есть $x_{112122}$. Оказывается, что операция $\tau_{S}$ согласуется со всеми остальными операциями обращения, обозначенными выше буквой $\tau$ с различными индексами.

Лемма 7. Инволючии изменения ориентации в пространствах $\mathscr{A}(p), \mathscr{B}(p)$, $S(p)$ и $U(p)$ коммутируют с четырьмя стрелками диаграммы в лемме 5. Точнее, имеет место коммутативный куб

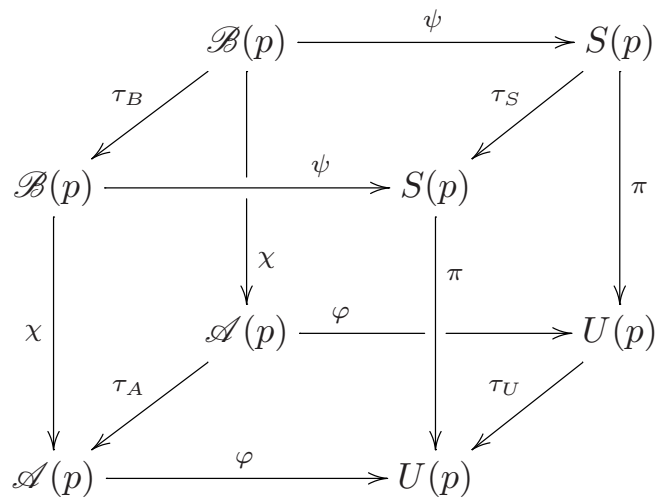

(напомним, ито $\mathfrak{g}=\mathfrak{g l}_{N}, S(p)=\left[S(\mathfrak{g})^{\otimes p}\right]^{\mathfrak{g}}$ u $U(p)=\left[U(\mathfrak{g})^{\otimes p}\right]^{\mathfrak{g}} ;$ все отображения были определены выше, в частности, $\pi$ - это изоморфизм ПуанкареБиркгофа-Bитта).

Доказательство. На самом деле, единственное, что еще не было доказано это коммутативность верхней грани куба. Поскольку все вертикальные стрелки - изоморфизмы, этот факт вытекает из коммутативности остальных пяти граней, которая была доказана ранее в различных местах статьи.

В силу коммутативности верхней грани куба для доказательства не- $\tau_{B}$-инвариантности какой-то диаграммы Якоби (элемента пространства $\mathscr{B}(p))$ достаточно показать не- $\tau_{S}$-инвариантность его $\psi$-образа в алгебре ожерелий. Поскольку минимальный порядок необратимого ожерелья равен 6 , а мы хотим найти необратимую диаграмму с нечетным числом ног, это число должно быть 
не менее 7. Оказывается, что существует ненулевая диаграмма, имеющая ровно 7 ног, и это дает второе доказательство теоремы.

Предложение 2. Следующая диаграмма отлична от 0 как элемент пространства $\mathscr{B}(2)$ :

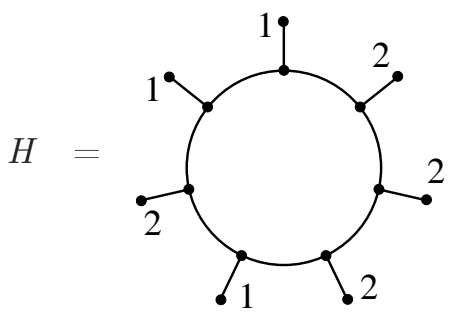

Доказательство. Прямое вычисление показывает, что образ этой диаграммы в алгебре $S(2)$ (для достаточно большого $N$ ), выраженный через ожерелья, равен

$$
N\left(x_{1121222}-x_{1122212}\right)+3 x_{2}\left(x_{112212}-x_{112122}\right),
$$

т. е. отличен от 0. Выражение для $\psi(H)$ содержит 128 слагаемых (по числу $2^{7}$ разрешений трехвалентных вершин), из которых только 8 содержат необратимые ожерелья, например

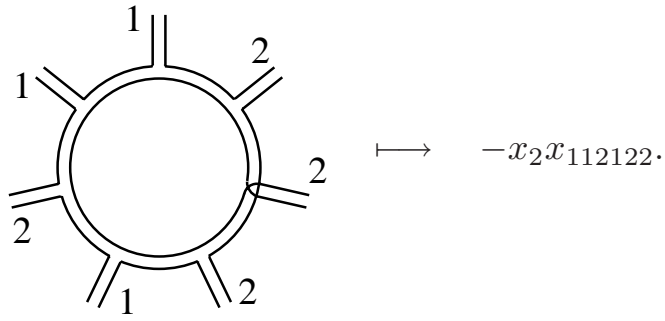

Этот результат также может быть получен с помощью компьютерной программы, которую можно найти на интернет-странице [5].

Замечание 1. Диаграмма $H$ была впервые обнаружена Д. Бар-Натаном: она фигурирует в файле table.m на его веб-сайте (см. [3]) как один из базисных элементов пространства двухцветных диаграмм. Однако в препринте [3], содержащем комментарии к этой таблице, сказано, что программа, при помощи которой она была получена, не готова к публикации по причине ее «запутанности» и ввиду некоторых недостатков в алгоритме.

Замечание 2. Из той же таблицы следует, что 7 - минимальная степень инварианта Васильева, способного различать ориентацию 2-компонентных струнных зацеплений. Необходимые для проверки вычисления можно провести вручную.

\section{§6. Переход к неоснащенному случаю}

Как упоминалось во введении, результаты, полученные в $\S 3$ и 5, на самом деле относятся к оснащенным зацеплениям, поскольку мы не принимали во внимание одночленные соотношения. Вообще говоря, определение ориентации оснащенного зацепления является более простой задачей, поскольку в теории с 
оснащениями есть дополнительная структура, которая (в принципе) может менять свои свойства при обращении. В этом параграфе мы докажем, что основная теорема остается верной и в случае обычных (неоснащенных) зацеплений.

Действительно, обозначим через $\mathscr{A}^{\prime}(2)$ фактор пространства $\mathscr{A}(2)$ по одночленным соотношениям, т. е. по идеалу, порожденному диаграммами $a_{1}$ (одна хорда, прикрепленная к первой компоненте носителя) и $a_{2}$ (соответственно одна хорда на второй компоненте носителя): $\mathscr{A}^{\prime}(2)=\mathscr{A}(2) /\left\langle a_{1}, a_{2}\right\rangle$, где угловые скобки обозначают двусторонний идеал с указанными в них образующими. Факторалгебра $\mathscr{A}^{\prime}(2)$ может быть вложена в $\mathscr{A}(2)$ как подалгебра: по структурной теореме для кокоммутативных алгебр Хопфа алгебра $\mathscr{A}(2)$ является универсальной обертывающей алгебры Ли своих примитивных элементов Р. Поэтому, если мы возьмем подпространство в $P$, порожденное всеми примитивными диаграммами, за исключением $a_{1}$ и $a_{2}$, мы получим вложение $\mathscr{A}^{\prime}(2) \subset \mathscr{A}(2)$. Поскольку диаграммы, фигурирующие в предложении 1, принадлежат этому подпространству, мы заключаем, что необратимость имеет место и для неоснащенных зацеплений.

Покажем теперь, что второе доказательство (§5) также проходит в неоснащенном случае. Пусть $\chi: \mathscr{A}(2) \rightarrow \mathscr{B}(2)$ - определенный выше изоморфизм.

Лемма 8. Подпространство $\chi^{-1}\left(\mathscr{A}^{\prime}(2)\right)=\mathscr{B}^{\prime}(2)$ совпадает с подалгеброй в $\mathscr{B}(2)$, порожденной всеми связными диаграммами Якоби, за исключением $b_{1}=\stackrel{\mathbf{1}}{\bullet} \mathbf{1} u b_{2}=\stackrel{\mathbf{2}}{\bullet} \mathbf{2}$.

Доказательство. Этот факт не вполне очевиден по той причине, что изоморфизм линейных пространств $\chi$ не является гомоморфизмом алгебр, а подалгебры $\mathscr{A}^{\prime}(2)$ и $\mathscr{B}^{\prime}(2)$ определяются своими образующими в смысле разных операций умножения. Тем не менее легко усмотреть непосредственно, что $\chi$-образ диаграммы Якоби, отличной от $b_{1}$ и $b_{2}$, Является линейной комбинацией произведений связных хордовых диаграмм, отличных от $a_{1}$ и $a_{2}$.

Лемма показывает, что семиногая диаграмма $H$, фигурирующая в предложении 2 , является элементом подалгебры $\mathscr{B}^{\prime}(2)$, ответственной за инварианты Васильева неоснащенных длинных зацеплений. Поскольку эта диаграмма не равна 0, существование инварианта седьмого порядка, различающего ориентацию, вторично доказано.

\section{§7. Открытые проблемы}

Вопрос 1. Существует ли гомоморфизм $\mathscr{B}(1) \rightarrow \mathscr{B}(2)$, образ которого не содержится в $\tau$-инвариантном подпространстве $\mathscr{B}(2)$ ?

Комментарий. Если бы такой гомоморфизм существовал, то можно было бы попытаться применить технику, разработанную в этой работе, к проблеме обратимости узлов. K сожалению, известный оператор удвоения $\Delta: \mathscr{B}(1) \rightarrow \mathscr{B}(2)$ (определенный в [1], [12]), как легко убедиться, для данной цели не подходит.

Вопрос 2. Возможно ли с помощью инвариантов Васильева заметить необратимость замкнутого 2-компонентного зацепления?

Комментарий. Комбинаторный объект, применяемый для описания инвариантов конечного типа замкнутых зацеплений - это пространство хордовых диаграмм на двух окружностях $\mathscr{A}\left(S_{2}^{1}\right)$. Ядро очевидного эпиморфизма $\mathscr{A}\left(\mathbb{R}_{2}^{1}\right)$ 
$\rightarrow \mathscr{A}\left(S_{2}^{1}\right)$ порождено так называемыми линк-соотношениями (см. [4]). Для решения вопроса необходимо разобраться во взаимосвязи линк-соотношений с операцией замены ориентации.

\section{Благодарности}

Мы благодарим А. С. Хорошкина, С. А. Локтева и Э. Б. Винберга за полезные сведения по теории инвариантов, а также С. К. Ландо и рецензента, прочитавших рукопись и сделавших ряд ценных замечаний.

\section{ЛитерАТУРА}

[1] D. Bar-Natan, On the Vassiliev knot invariants, Topology, 34:2 (1995), 423-472.

[2] D. Bar-Natan, Vassiliev homotopy string link invariants, J. Knot Theory Ramifications, 4:1 (1995), 13-32.

[3] D. Bar-Natan, Some computations related to Vassiliev invariants, http://www.math. toronto.edu/ drorbn/papers, 1996.

[4] D. Bar-Natan, S. Garoufalidis, L. Rozansky, D. Thurston, The Arhus integral of rational homology 3-spheres II: Invariance and universality, Selecta Math. (N. S.), 8:3 (2002), 341-371.

[5] С. Дужин, Программы и файлы данных, относящиеся $к$ вычислению весовых систем $\varphi$ u $\psi$, http://www.pdmi.ras.ru/ arnsem/dataprog/OrLinks/.

[6] S. Chmutov, S. Duzhin, J. Mostovoy, CDBook. Introduction to Vassiliev knot invariants, draft version of a book, Online at http://www.pdmi.ras.ru/ duzhin/papers/.

[7] T. Fiedler, Isotopy invariants for closed braids and almost closed braids via loops in stratified spaces, http://arxiv.org/math.GT/0606443.

[8] A. Kawauchi, The invertibility problem on amphicheiral excellent knots, Proc. Japan Acad. Ser. A Math. Sci., 55:10 (1979), 399-402.

[9] M. Kontsevich, Vassiliev's knot invariants, Adv. Soviet Math., 16, Part 2 (1993), $137-150$.

[10] X.-S. Lin, Finite type link invariants and the invertibility of links, Math. Res. Lett., 3:3 (1996), 405-417; http://arxiv.org/q-alg/9601019.

[11] X.-S. Lin, Finite type link-homotopy invariants, Enseign. Math. (2), 47:3-4 (2001), 315-327; http://arxiv.org/math.GT/0012095.

[12] T. Q. T. Le, J. Murakami, The universal Vassiliev-Kontsevich invariant for framed oriented links, Compositio Math., 102:1 (1996), 41-64.

[13] T. Stanford, Finite-type invariants of knots, links and graphs, Topology, 35:4 (1996), 1027-1050.

[14] H. F. Trotter, Non-invertible knots exist, Topology, 2:4 (1963), 275-280.

[15] Э. Б. Винберг, В. Л. Попов, Теория инвариантов, в кн.: Итоги науки и техники, Современные проблемы математики, Фундаментальные направления, т. 55, ВИНИТИ, М., 1989, 137-314.

[16] P. Vogel, Algebraic structures on modules of diagrams, Institut de Mathématiques de Jussieu, Prépublication 32, August 1995; Revised in 1997, http://www.math.jussieu. $\mathrm{fr} / \sim$ vogel/.

[17] Г. Вейль, Классические группь, их инварианты и представления, ИЛ, М., 1947.

С.-Петербургское отделение

Математического института им. В. А. Стеклова

Поступило в редакцию

e-mail: duzhin@pdmi.ras.ru

11 октября 2005 г.

С.-Петербургское отделение

Математического института им. В. А. Стеклова

e-mail: max.karev@gmail.com 